Received: 12.08 .2019

Revised: 15.10 .2019

Accepted: 25.10 .2019

DOI: $10.17804 / 2410-9908.2019 .5 .060-072$

\title{
THE EFFECT OF DAMAGE AT AN ENSEMBLE OF MICROSTRUCTURE POINTS ON THE MARGIN OF SAFETY IN STRUCTURALLY HETEROGENEOUS MATERIALS
}

\author{
S. S. Volkov \\ Institute of Engineering Science, Urals Branch of the Russian Academy of Sciences, \\ 34 Komsomolskaya St., Ekaterinburg, 620219, Russian Federation \\ https://orcid.org/0000-0002-6481-5052 volkovss48@yandex.ru \\ Corresponding author. E-mail: volkovss48@yandex.ru \\ Address for correspondence: ul. Komsomolskaya, 34, Ekaterinburg, 620049, Russian Federation \\ Tel.: +7 (343)371 7728
}

Fracture concentration regions are considered in a microstructure under loading. A mathematical model of a micro-heterogeneous medium with random properties of elements is used for the calculations. The initial data in the problem includes the characteristics of the distributions of random elastic moduli and ultimate strengths in the microstructure elements. The microstructure strength condition is a difference between stresses and ultimate strengths for a multitude of points of a given configuration. The probability of stress simultaneously exceeding the ultimate strength in a given set of elements determines the probability of fracture in this ensemble of points and relative damage at the micro level. A multidimensional normal distribution is used to calculate damage. The structure of the correlation matrix of distribution takes into account the kind of the damage concentration region. The relationship between critical microstructure damage and the margin of safety is determined. Examples of calculating the probability of fracture in two, three, and four microstructure elements are given.

Keywords: random properties, microstructure damage, multidimensional probability distribution, ultimate strength, margin of safety.

\section{References}

1. Yokobori T. An Interdisciplinary Approach to Fracture and Strength of Solids, Groningen, Wolters-Noordhoff Scientific LTD, 1968.

2. Vildeman V.E., Sokolkin Yu.V., Tashkinov A.A. Mekhanika neuprugogo deformirovaniya i razrusheniya kompozitsionnykh materialov [Mechanics of Non Elastic Deformation and Fracture of Composite Materials]. Nauka Publ., Moscow, 1997. (In Russian).

3. Volkova T.A., Volkov S.S. Microstructure damage related to stress- strain curve for grain composites. Theoretical and Applied Fracture Mechanics, vol. 52, iss. 2, 2009, pp. 83-90. DOI: 10.1016/j.tafmec.2008.02.004.

4. Sih G. C. Fracture mechanics in retrospect in contrast to multiscaling in prospect. In: Proceedings of the 17-th National Conference of Italian Group of Fracture, ed. by A. Finelli and L. Nobile, Bologna, June 16-18, 2004, pp. 15-37.

5. Trusov P.V., Volegov P.S., Yanz A.Yu. Two-scale models of polycrystals: Evaluation of validity of Ilyushin's isotropy postulate at large displacement gradients. Physical Mesomechanics, 2016, vol. 19, no. 1, pp. 21-34. DOI: 10.1134/S1029959916010033. 
6. Surikova N.S., Panin V.E., Derevyagina L.S., Lutfullin R.Ya., Manzhina E.V., Kruglov A.A., Sarkeeva A.A. Micromechanisms of deformation and fracture in a VT6 titanium laminate under impact load. Physical Mesomechanics, 2015, vol. 18, no. 3, pp. 250-260. DOI: 10.1134/S1029959915030091.

7. Schastlivtsev V.M., Tabatchikova T.I., Yakovleva I.L., Klyueva S.Yu., Kruglova A.A., Khlusova E.I., Orlov V.V. Microstructure and properties of low-carbon weld steel after thermomechanical strengthening. The Physics of Metals and Metallography, 2012, vol. 113, no. 5, pp. 480488. DOI: 10.1134/S1029959915030091.

8. Pugacheva N.B., Bykova T.M., Trushina E.B., Malygina I.Yu. The Structural State and Properties of a Deposited Coating for an Internal Combustion Engine Valve. Diagnostics, Resource and Mechanics of materials and structures, 2018, iss. 5, pp. 74-85. DOI: 10.17804/24109908.2018.5.074-085. URL: http://dream-journal.org/issues/2018-5/2018-5_186.html (accessed 30.11.2019).

9. Zaitsev A.V. Second-order moment functions for the random structure of unidirectionally reinforced fibrous composites. In: Vestnik UGTU-UPI. Mekhanika microneodnorodnykh materialov $i$ razrushenie [Herald of UGTU-UPI, Mechanics of Micro-Heterogeneous Materials and Fracture]. Ekaterinburg, UGTU-UPI Publ., 2006, no. 11 (82), pp. 161-167. (In Russian).

10. Smirnov S.V., Konovalov A.V., Myasnikova M.V., Khalevitsky Yu.V., Smirnov A.S., Igumnov A.S. A Computational Model of V95/sicp (7075/ Sicp) Aluminum Matrix Composite Applied to Stress-Strain State Simulation under Tensile, Compressive and Shear Loading Conditions. Diagnostics, Resource and Mechanics of materials and structures, 2017, iss. 6, pp. 16-27. DOI: 10.17804/2410-9908.2017.6.016-027. Available at: http://dream-journal.org/issues/20176/2017-6_133.html (accessed 14.10.2019).

11. Panin V.E., Derevyagina L.S., Lebedev M.P., Syromyatnikova A.S., Surikova N.S., Pochivalov Yu.I., Ovechkin B.B. Scientific Basis for Cold Brittleness of Structural BCC Steels and Their Structural Degradation at Below Zero Temperatures. Phys. Mesomech., 2017, vol. 2 (2), pp. 125-133. DOI: 10.1134/S1029959917020023.

12. Panin S.V., Marushchak P.O., Vlasov I.V., Eremin A.V., Byakov A.V., Syromyatnikova A.S., Stankevich R. Structure and Fatigue Durability of 09mn2si Pipe Steel after Long-Term Operation in Far North Conditions. Diagnostics, Resource and Mechanics of materials and structures, 2017, iss. 4, pp. 81-85. DOI: 10.17804/2410-9908.2017.4.081-085. Available at: http://dreamjournal.org/issues/2017-4/2017-4_164.html (accessed 14.10.2019).

13. Mironov V.I., Emelyanov I.G., Vichuzhanin D.I., Kamantsev I.S., Yakovlev V.V., Ogorelkov D.A., Zamaraev L.M. A Method for Experimental Investigation of Degradation Processes in Materials. Diagnostics, Resource and Mechanics of materials and structures, 2019, iss. 2, pp. 16-27. DOI: 10.17804/2410-9908.2019.2.016-027. Available at: http://dream-journal.org/issues/2019-2/20192_246.html (accessed 30.11.2019).

14. Mitropolsky A.K. Tekhnika statisticheskykh vychisleniy [The technique of Statistical Computations]. Nauka Publ., Moscow, 1971. (In Russian).

15. Volkova T.A., Volkov S.S. Microstructure damage at ensemble of points for grain composites. Theoretical and Applied Fracture Mechanics, 2010, vol. 54, iss. 3, pp. 149-155. DOI: $10.1016 /$ j.tafmec.2010.10.010.

16. McLean D. Mechanical Properties of Metals, Wiley, New York, 1962. 
Подана в журнал: 12.08.2019

УДК 539.3

DOI: $10.17804 / 2410-9908.2019 .5 .060-072$

\title{
ВЛИЯНИЕ ПОВРЕЖДЕННОСТИ АНСАМБЛЯ ТОЧЕК МИКРОСТРУКТУРЫ НА ЗАПАС ПРОЧНОСТИ СТРУКТУРНО-НЕОДНОРОДНЫХ МАТЕРИАЛОВ
}

\author{
С. С. Волков \\ Федеральное государственное бюджетное учреждение науки \\ Институт машиноведения Уральского отделения Российской академии наук \\ д. 34, ул. Комсомольская, 620049, Екатеринбург, Российская Федерация \\ https://orcid.org/0000-0002-6481-5052 volkovss48@yandex.ru \\ Ответственный автор. Электронная почта: volkovss48@yandex.ru \\ Адрес для переписки: ул. Комсомольская 34, Екатеринбург, Российская Федерация \\ Тел.: +7(343) 371-77-28
}

Рассматриваются зоны концентрации разрушений в элементах микроструктуры деформируемого материала. Для расчетов используется математическая модель микронеоднородной среды со случайными свойствами элементов. Исходными данными задачи являются характеристики распределения случайных модулей упругости и предела прочности в элементах микроструктуры. Микроструктурное условие прочности представляет собой разницу между напряжениями и пределом прочности для множества точек данной конфигурации. Вероятность одновременного превышения напряжением предела прочности в данном множестве элементов определяет вероятность разрушения этого ансамбля точек и относительную поврежденность на микроуровне. В расчетах поврежденности используется многомерное нормальное распределение. Структура корреляционной матрицы распределения учитывает вид зоны концентрации разрушений. Найдена зависимость между величиной критической микроструктурной поврежденности материала и запасом прочности. Проведены примеры расчетов вероятности разрушения в двух, трех и четырех элементах микроструктуры.

Ключевые слова: случайные свойства, поврежденность микроструктуры, многомерное распределение вероятностей, предел прочности, запас прочности.

\section{1. Введение}

Под действием нагрузки конструкционные материалы накапливают поврежденность элементов микроструктуры. На ранних стадиях микроразрушение имеет рассеянный характер. Затем увеличивается вероятность одновременного разрушения в близких точках. Постепенно формируются зоны повреждения, содержащие несколько элементов микроструктуры. Слияние таких зон приводит к развитию магистральной трещины и потере несущей способности конструкции. Критическая поврежденность материала определяется из эксперимента и инженерного опыта и зависит, прежде всего от степени ответственности конструкции. Необоснованное увеличение запаса прочности снижает качество конструкции, увеличивает ее вес и размеры. Изучение процесса разрушения элементов микроструктуры дает возможность обосновать величину запаса прочности материала.

В задачах механики структурно-неоднородных сред рассматриваются прочностные и деформационные свойства материала на нескольких уровнях структуры, включая микро- и нано- уровни [1-8]. Характеристики случайных свойств микроструктуры используются при моделировании вероятностного процесса деформирования и разрушения материала в конструкциях $[2,3,9,10]$. Проводится анализ формирования зон внутреннего разрушения [10, 
11], в том числе для магистральных трубопроводов при длительной эксплуатации [12]. Выполняются экспериментальные исследования процессов деградации материалов с учетом их структуры [13].

При полной постановке стохастической краевой задачи механики микронеоднородных сред требуется найти многоточечные законы распределения случайных деформаций и напряжений в элементах микроструктуры. Такие законы дают исчерпывающее решение задачи в рамках теории случайных функций $[2,3,14]$. Они используются для построения статистических теорий деформирования и оценки надежности материалов и элементов конструкций. Для расчета случайных микроструктурных напряжений и деформаций используются данные о свойствах микроструктуры композита. Случайные упругие и прочностные свойства микроструктуры определяются в общем случае многоточечными моментными функциями. Эти функции находятся с помощью экспериментальных распределений, полученных при изучении химического состава, пористости, размеров и формы включений $[2,9]$.

В работе [3] сделано обобщение методов расчета микроструктурных напряжений для зернистых композитов с непрерывным распределением свойств микроструктуры. В частности, такими материалами являются металлы и порошковые композиты. Исследована точность метода. Показана возможность его использования для материалов с непрерывным распределением и большими вариациями свойств микроструктуры. Получены расчетные формулы $n$-точечных моментных функций моментных функций произвольного порядка для микронапряжений, а также более детальные формулы корреляционных функций напряжений в зернистых композитах.

Для расчета многоточечных условий прочности применяются многоточечные законы распределения микронапряжений. Микроструктурное условие прочности представляет собой разницу между случайным эквивалентным напряжением и случайным пределом прочности. Для оценки вероятности одновременного разрушения в нескольких точках нужно найти соответствующую многоточечную плотность распределения микроструктурного условия прочности. Это позволяет вычислить относительное содержание микроскопических зон разрушения данной конфигурации [15]. Для прогнозирования ранних стадий разрушения и оценки прочности материала исследуется вероятность многоточечного разрушения микроструктуры.

\section{2. Корреляционные функции микроструктурных напряжений}

Для макроизотропных стохастических зернистых композитов рассмотрим задачу механики микронеоднородных сред, связывающую случайные тензоры модулей упругости $\Theta(X)$, тензоры деформаций $\boldsymbol{\varepsilon}(X)$ и напряжений $\sigma(X)$ в элементах микроструктуры $X=\left(x_{1}, x_{2}\right.$, $\left.x_{3}\right)$. Обозначим угловыми скобками математическое ожидание случайной величины. Предполагается, что макродеформации $\mathbf{e}=\langle\boldsymbol{\varepsilon}(X)>$ найдены при решении краевой задачи для данной конструкции. Заданы распределение случайного модуля Юнга $E(X)$ и детерминированный коэффициент Пуассона v. Используя законы распределения случайных свойств микроструктуры, нужно найти параметры распределения случайных микроструктурных деформаций и напряжений.

Расчет моментных функций для деформаций $\varepsilon(X)$ выполняется с помощью интегрального операторного уравнения, использующего вторые производные тензора Грина $[2,3]$. Это решение содержит произведения вариаций случайных тензоров модулей упругости $\Theta(X), \Theta(Y), \ldots \Theta(Z)$ во всем ансамбле исследуемых точек $X, Y, \ldots Z$. Для материалов, не содержащих начальных микроразрушений, может быть принято предположение о статистической независимости свойств микроструктуры в различных точках или взаимной независимости полей деформаций на разных этапах метода приближений [3]. В более общем случае нужно использовать данные эксперимента о совместном распределении свойств среды во множестве точек. Это дает возможность учесть степень упорядоченности микроструктуры, 
форму включений, характер микроповрежденности. Для расчета структурных напряжений $\sigma$ $(X)$, используется свертка тензоров по двум индексам «••»:

$$
\boldsymbol{\sigma}(X)=\Theta(X) \cdots \boldsymbol{\varepsilon}(X)=\Theta(X) \cdot\left(\mathbf{e}+\boldsymbol{\varepsilon}^{\circ}(X)\right) .
$$

Методы расчета корреляционных функций напряжений $K_{\sigma}(r)$ приведены в работе [3]. При этом используется корреляционная функция модуля Юнга $K_{E}(r)=\left\langle E^{\circ}(X) E^{\circ}(Y)\right\rangle$ для точек на расстоянии $r=|X-Y|$. В приближенном варианте корреляционная функция $K_{\sigma}(r)$ напряжений имеет вид

$$
K_{\sigma}(r)=e^{2} \frac{4}{9 E^{2}(1-v)^{2}}\left(E^{2} K_{E}(r)(0,8-v)^{2}+0,5(1,4-v)^{2} K_{E}(r)^{2}\right) .
$$

Корреляционные функции составляющих тензора напряжений становятся основой расчета корреляционных матриц [15] при построении совместного распределения напряжений в ансамбле точек $X, Y, \ldots Z$.

\section{3. Микроструктурное условие прочности}

На предел прочности элементов микроструктуры существенно влияют размер зерна, распределение легирующих добавок, начальная и приобретенная пористость. Перечисленные параметры для реальных материалов на уровне микроструктуры имеют случайный характер. Следовательно, микроструктурный предел прочности $S(X)$ является случайной величиной. Параметры его распределения можно найти по данным эксперимента, используя функции от случайных величин. Так, например, с ростом величины зерна металла предел прочность при отрыве уменьшается [16]. По анализу микрошлифов находим распределение диаметров зерен и затем вычисляем плотность распределения пределов прочности. Известны также данные о влиянии легирующих добавок на сопротивление металлов отрыву и срезу [16].

Микроструктурное условие прочности (функция прочности) $w(X)$ в точке $X$ представляет собой разницу между случайным эквивалентным напряжением $\sigma(X)$ и случайным пределом прочности $S(X)$ [3]. Случайная величина $\sigma(X)$ может быть интенсивностью напряжений или одной из составляющих тензора напряжений в зависимости от вида нагрузки:

$$
w(X)=\sigma(X)-S(X)
$$

Если задан ансамбль точек микроструктуры $X_{1}, X_{2}, \ldots X_{n}$, то микроструктурное условие прочности запишем в виде случайного вектора:

$$
\mathbf{W}=\left\{w\left(X_{1}\right), \ldots w\left(X_{n}\right)\right\} .
$$

Если параметр $w\left(X_{i}\right)>0$, то напряжение в точке $X_{i}$ больше предела прочности, поэтому в элементе микроструктуры произойдет разрушение. При $w\left(X_{i}\right)<0$ разрушение не происходит, так как напряжение находится в допустимой области безопасных значений. Одновременное разрушение в точках ансамбля произойдет, если все координаты вектора условия прочности $\mathbf{W}$ положительны. Вероятность такого разрушения равна многомерному интегралу от плотности распределения $f_{w}\left(t_{1}, t_{2}, \ldots t_{n}\right)$ случайного вектора $\mathbf{W}$. Областью интегрирования будут интервалы от нуля до бесконечности по всем $n$-измерениям: 


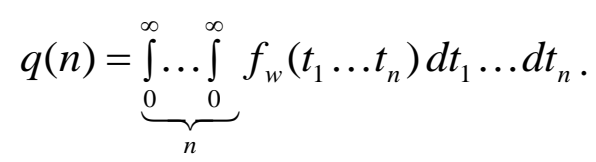

По микроструктурному условию прочности $\mathbf{W}$ оценивается поврежденность $q(n)$, т. е. относительное содержание микроскопических зон разрушения заданной конфигурации.

Рассмотрим условие прочности в двух точках $X$ и $Y$. Если напряжения $\sigma(X)$ и предел прочности $S(X)$ распределены нормально, то коэффициент вариации $k_{w}$ и коэффициент корреляции $\rho_{w}(r)$ случайной величины $w(X)$ вычисляются по следующим формулам:

$$
\begin{gathered}
k_{w}=\frac{\sqrt{<\sigma^{o}(X)^{2}>+<S^{o}(X)^{2}>}}{|<\sigma(X)>-<S(X)>|} ; \\
\rho_{w}(r)=\frac{<w^{o}(X) w^{o}(Y)>}{<w^{o}(X)^{2}>}=\frac{<\sigma^{o}(X) \sigma^{o}(Y)>+<S^{o}(X) S^{o}(Y)>}{<\sigma^{o}(X)^{2}>+<S^{o}(X)^{2}>} .
\end{gathered}
$$

Рассмотрим нормальную $n$-мерную плотность распределения $f_{w}\left(t_{1}, t_{2}, \ldots t_{n}\right)$ [14]. Параметры этой функции определяются математическим ожиданием $m$, коэффициентом вариации $k$ микроструктурного условия прочности $w(X)$ и матрицей коэффициентов корреляции $\mathbf{P}=$ $\left[\rho_{\mathrm{ij}}\right]$ для случайных параметров $w\left(X_{1}\right),\left(X_{2}\right), \ldots w\left(X_{n}\right)$. В расчетах используется обратная матрица коэффициентов корреляции $\Lambda$ :

$$
\begin{gathered}
f_{w}\left(t_{1} \ldots t_{n}\right)=\frac{1}{\sqrt{(2 \pi)^{n}} \cdot k^{n} m^{n} \sqrt{\Delta}} \exp \left(-\frac{1}{2 \Delta} \sum_{i} \sum_{j} \Lambda_{i j} \frac{\left(t_{i}-m\right)\left(t_{j}-m\right)}{k^{2} m^{2}}\right) \\
m=<w(X)>; k^{2}=\frac{<w^{o}(X)^{2}>}{m^{2}} ; \rho_{i j}=\frac{<w^{o}\left(X_{i}\right) w^{o}\left(X_{j}\right)>}{<w^{o}(X)^{2}>} ; \\
\mathbf{P}=\left[\rho_{i j}\right], \Delta=\operatorname{det} \mathbf{P}, \Lambda=\mathbf{P}^{-1}
\end{gathered}
$$

Для расчета интеграла (5) выполним замену координат $\frac{t_{i}-m}{k|m|}=x_{i}$. Область интегрирования $(0, \infty)$ перейдет при этом в область $\left(k^{-1}, \infty\right)$. Заметим, что математическое ожидание условия прочности $m$ отрицательно, так как для работающих конструкций среднее напряжение меньше предела прочности. В результате вероятность одновременного микроразрушения в $n$-точках примет вид:

$$
q(l, k, P)=\int_{k^{-1}}^{\infty} \ldots \int_{k^{-1}}^{\infty} f_{w}\left(t_{1} \ldots t_{l}\right) d x_{1} \ldots d x_{l} .
$$

Корреляционные функции составляющих тензора напряжений и микроструктурного предела прочности становятся основой для построения совместного распределения условия прочности в исследуемых точках. Структура корреляционной матрицы $\mathbf{P}=\left[\rho_{i j}\right]$ отражает 
конфигурацию исследуемых ансамблей точек. Корреляция $\left.\rho_{i j}(r)=\rho\left|X_{i}-X_{j}\right|\right)$ зависит от близости точек $X_{i}$ и $X_{j}$. Чем дальше точки, тем меньше корреляция. Рост корреляции в удаленных точках отражает также характер развития микроповрежденности. При этом прослеживается переход от стадии рассеянного и независимого разрушения отдельных элементов микроструктуры к этапу концентрации поврежденностей и подготовки условий для развития магистральной трещины. Математическое ожидание $m=\langle w(X)>$ оценивает удаленность нагрузки от опасных значений. Коэффициент вариации $k$ характеризует однородность материала на уровне микроструктуры. Чем больше коэффициент вариации, тем менее однородна микроструктура.

\section{4. Разрушение в двух точках}

Пусть точки микроструктуры $X$ и $Y$ расположены на расстоянии $r$. Для напряжений $\sigma(X)$ и $\sigma(Y)$ задана совместная плотность распределения вероятности $z=f_{\sigma}(x, y)$. Вектор $\mathbf{W}$ условия прочности (4) будет следующим:

$$
\mathbf{W}=\{w(X), w(Y)\}=\{\sigma(X)-S(X), \sigma(Y)-S(Y)\} .
$$

Нормальная плотность $f(x, y)$ совместного распределения $w(X), w(Y)$ зависит от параметров $m, k, \rho$.

$$
m=\left\langle w(X)>=\left\langle w(Y)>; k=\frac{\sqrt{<w^{\mathrm{O}}(X)^{2}>}}{m} ; \rho=\frac{<w^{\mathrm{o}}(X) w^{\mathrm{O}}(Y)>}{\left.<w^{\mathrm{o}}(X)^{2}\right\rangle} .\right.\right.
$$

Запишем матрицы $\mathbf{P}$ и $\boldsymbol{\Lambda}$ :

$$
P=\left[\begin{array}{cc}
1 & \rho \\
\rho & 1
\end{array}\right] ; \quad \Lambda=\frac{1}{\Delta}(1-\rho)\left[\begin{array}{cc}
1 & -\rho \\
-\rho & 1
\end{array}\right] ; \quad \Delta=1-\rho^{2} .
$$

Перейдем к расчету вероятности разрушения $q=q(k, \rho)$ в двух точках $X$ и $Y$. В соответствии с формулой (11) вероятность разрушения зависит от двух параметров условия прочности: $k$ - коэффициента вариации и $\rho$ - коэффициента корреляции для точек $X, Y$ :

$$
q(k, \rho)=\int_{k^{-1}}^{\infty} \int_{k^{-1}}^{\infty} \frac{1}{2 \pi \sqrt{1-\rho^{2}}} \exp \left(-\frac{x^{2}-2 \rho x y+y^{2}}{2\left(1-\rho^{2}\right)}\right) d x d y .
$$

\section{5. Разрушение в трех точках}

Вероятность разрушения в трех точках $X, Y, Z$ определяется плотностью совместного распределения условия прочности $f(x, y, z)$ и вычисляется по формуле (11) при $l=3$.

$$
q=\int_{k^{-1}}^{\infty} \int_{k^{-1}}^{\infty} \int_{k^{-1}}^{\infty} f(x, y, z) d x d y d z
$$

Пусть точки расположены в вершинах равностороннего треугольника со сторонами $r=|X-Y|=|X-Z|=|Y-Z|$. Коэффициент корреляции $\rho=\rho(r)$ зависит от расстояния между точками. Корреляционная матрица $\mathbf{P}$ и обратная к ней матрица $\boldsymbol{\Lambda}$ имеют следующий вид: 


$$
\mathbf{P}=\left[\begin{array}{lll}
1 & \rho & \rho \\
\rho & 1 & \rho \\
\rho & \rho & 1
\end{array}\right], \quad \boldsymbol{\Lambda}=(1-\rho)\left[\begin{array}{ccc}
1+\rho & -\rho & -\rho \\
-\rho & 1+\rho & -\rho \\
-\rho & -\rho & 1+\rho
\end{array}\right], \quad \Delta=(1-\rho)^{2}(1+2 \rho)
$$

Функция $f(x, y, z)$ будет иметь вид:

$$
\begin{aligned}
& f(x, y, z)= \\
& =\frac{1}{\sqrt{8 \pi^{3}}(1-\rho) \sqrt{1+2 \rho}} \exp \left(-\frac{(1+\rho)\left(x^{2}+y^{2}+z^{2}\right)-2 \rho(x y+x z+y z)}{2(1-\rho)(1+2 \rho)}\right)
\end{aligned}
$$

\section{6. Разрушение в четырех точках}

Если четыре точки находятся в вершинах тетраэдра, расстояния между ними будут одинаковыми. Это определит структуру корреляционной матрицы.

$$
\begin{gathered}
\mathbf{P}=\left[\begin{array}{cccc}
1 & \rho & \rho & \rho \\
\rho & 1 & \rho & \rho \\
\rho & \rho & 1 & \rho \\
\rho & \rho & \rho & 1
\end{array}\right] ; \quad \Lambda=(1-\rho)^{2}\left[\begin{array}{cccc}
1+2 \rho & -\rho & -\rho & -\rho \\
-\rho & 1+2 \rho & -\rho & -\rho \\
-\rho & -\rho & 1+2 \rho & -\rho \\
-\rho & -\rho & -\rho & 1+2 \rho
\end{array}\right] . \\
\Delta=(1-\rho)^{3}(1+2 \rho) .
\end{gathered}
$$

В соответствии с формулами $(8,11)$ получим следующую совместную плотность распределения условия прочности:

$f(x, y, z, u)=\frac{1}{4 \pi^{2} \cdot \Delta} \exp \left(-\frac{1}{2(1-\rho)(1+2 \rho)}\left((1+3 \rho)\left(x^{2}+y^{2}+z^{2}+u^{2}\right)-\rho(x+y+z+u)^{2}\right)\right)$.

Вероятность разрушения в четырех точках в вершинах тетраэдра определяется плотностью распределения $f(x, y, z, u)$ и вычисляется по формуле (11) при $l=4$ :

$$
q=\int_{k^{-1}}^{\infty} \int_{k^{-1}}^{\infty} \int_{k^{-1}}^{\infty} \int_{k^{-1}}^{\infty} f(x, y, z, u) d x d y d z d u
$$

В работе [15] исследовано влияние параметров распределения микроструктурной функции прочности на вероятность разрушения для различной конфигурации ансамбля точек микроструктуры. Так с ростом параметров $k$ и $\rho$ происходит наращивание поврежденности. Если оба параметра малы, поврежденность практически нулевая. С приближением хотя бы одного из параметров к его критическому значению вероятность повреждения вначале медленно, а затем заметно возрастает. Если оба параметра $k$ и $\rho$ находятся в диапазоне больших значений, поврежденность развивается лавинообразно. Характер развития поврежденности близок для всех ансамблей точек. При этом существенно отличается скорость приращения поврежденности в зависимости от конфигурации точек. Быстрее всего она возрастает в одной точке, затем медленнее в двух соседних точках. Одновременное разрушение в вершинах тетраэдра происходит быстрее, чем в линии из четырех точек. С ростом нагрузки и увеличением поврежденности меняются свойства материала, возрастает зависимость уда- 
ленных точек, увеличивается разброс свойств микроструктуры. Это приводит в свою очередь к еще более интенсивному росту поврежденности на следующих этапах нагружения конструкции.

С увеличением числа точек в зоне разрушения будет увеличиваться размерность корреляционных матриц. При этом уменьшается вероятность их одновременного разрушения. Критерием макроскопического разрушения материала служит его предельная поврежденность. Как правило, такая поврежденность возникает при переходе диаграммы деформирования к ниспадающей ветви [3].

\section{7. Коэффициент запаса прочности}

Отношение предела прочности материала $\sigma_{\text {в }}$ к напряжению в детали конструкции $\sigma$ называется коэффициентом запаса прочности: $[n]=\frac{\sigma_{B}}{\sigma}$. Эта величина является макроскопической характеристикой материала для данной конструкции. Рассматривается допустимое значение коэффициента запаса прочности $[n]$. Рекомендуемый диапазон изменения $[n]$ зависит от степени ответственности конструкции и условий, при которых были получены экспериментальные данные о работе детали в конструкции. Если были использованы приближенные экспериментальные данные о прочности детали и режимах ее нагружения, параметр $[n]$ должен быть больше. Если экспериментальные данные достаточно надежны, тогда $[n]$ можно уменьшить. Учет вероятностного рассеивания экспериментальных данных также дает возможность уменьшить $[n]$.

Рассмотрим случайные напряжения $\sigma(X)$, случайные пределы прочности $S(X)$ в элементах микроструктуры и их математические ожидания $\sigma=<\sigma(X)>; \sigma_{\mathrm{B}}=\langle S(X)>$. Пусть случайные величины $\sigma(X)$ и $S(X)$ независимы и имеют нормальные распределения вероятностей. Вычислим коэффициент вариации $k$ и коэффициент корреляции $\rho(r)$ случайной функции прочности $w(X)$ через параметры распределения случайных величин $\sigma(X)$ и $S(X)$. Введем в расчеты коэффициенты вариации $k_{\sigma}$ напряжений и $k_{\mathrm{B}}$ предела прочности. Преобразуя формулу (6), найдем связь между коэффициентом запаса прочности $[n]$, коэффициентами вариации соответствующих случайных величин $w(X), \sigma(X), \sigma_{\mathrm{B}}(X)$.

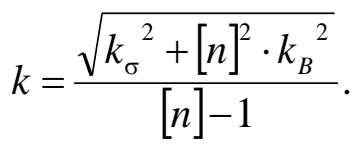

Рассмотрим также коэффициенты корреляции $\rho_{\sigma}=\rho_{\sigma}(X, Y)$ напряжений и $\rho_{B}=\rho_{B}(X, Y)$ пределов прочности. Преобразуем формулу (7):

$$
\rho(r)=\rho(|X-Y|)=\frac{\frac{\left(\sigma^{\mathrm{o}}(X) \sigma^{\mathrm{o}}(Y)>\right.}{<\sigma^{\mathrm{o}}(X)^{2}>} \frac{<\sigma^{\mathrm{o}}(X)^{2}>}{\sigma^{2}}+\frac{<S^{\mathrm{o}}(X) S^{\mathrm{o}}(Y)>}{<S^{\mathrm{o}}(X)^{2}>} \frac{<S^{\mathrm{o}}(X)^{2}>\sigma_{B}{ }^{2}}{\sigma_{B}{ }^{2}} \frac{\sigma^{2}}{\sigma^{2}}}{\frac{<\sigma^{\mathrm{o}}(X)^{2}>}{\sigma^{2}}+\frac{\sigma_{B}{ }^{2}<S^{\mathrm{o}}(X)^{2}>}{\sigma^{2}}} .
$$

В результате получим зависимость коэффициента корреляции функции прочности $\rho(r)$ от коэффициента запаса прочности материала $[n]$ :

$$
\rho(r)=\frac{\rho_{\sigma}(r) k_{\sigma}^{2}+[n]^{2} \rho_{B}(r) k_{B}^{2}}{k_{\sigma}^{2}+[n]^{2} k_{B}^{2}} .
$$


Формулы $(23,25)$ показывают также связь между величиной $[n]$ и параметрами распределения случайных деформационных и прочностных свойств элементов микроструктуры.

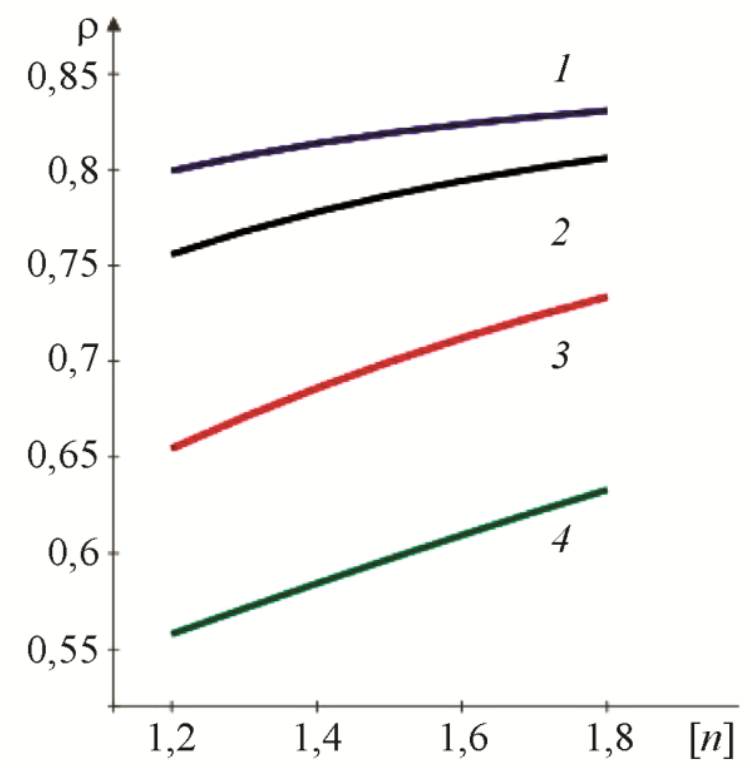

Рис. 1. Зависимость коэффициента запаса прочности $[n]$ и коэффициента корреляции $\rho$ при изменяющемся соотношении коэффициентов вариации $k_{\sigma} / k_{w}$.

Параметры линий 1, 2, 3, 4 указаны в тексте

На рис. 1 показана связь между коэффициентом корреляции функции прочности $\rho$ и коэффициентом запаса прочности $[n]$ при постоянных значениях параметров $\rho_{\sigma}=0,5$; $\rho_{B}=0,8$ и изменяющемся соотношении коэффициентов вариации: 1) $\left.k_{\sigma} / k_{w}=0,5 ; 2\right) k_{\sigma} / k_{w}=0,7$; 3) $k_{\sigma} / k_{w}=1,2$; 4) $k_{\sigma} / k_{w}=2$.

На рис. 2 представлено влияние параметров $\rho_{\sigma}, \rho_{B}$ связь между коэффициентом корреляции функции прочности $\rho$ и коэффициентом запаса прочности $[n]$. На рис. $2 a$ постоянны значения корреляции напряжений $\rho_{\sigma}=0,6$ и соотношение $k_{\sigma} / k_{w}=2$, при этом меняются значения корреляции пределов микроструктуры: 1) $\rho_{B}=1$; 2) $\rho_{B}=0,9$; 3) $\rho_{B}=0,8$;4) $\rho_{B}=0,7$. На рис. $2 \sigma$ постоянны значения корреляции пределов микроструктуры $\rho_{B}=0,6$ и соотношение $k_{\sigma} / k_{w}=1,2$, при этом меняются значения корреляции напряжений: 1) $\rho_{\sigma}=1$; 2) $\rho_{\sigma}=0,9$; 3) $\rho_{\sigma}=0,8$; 4) $\rho_{\sigma}=0,7$. 


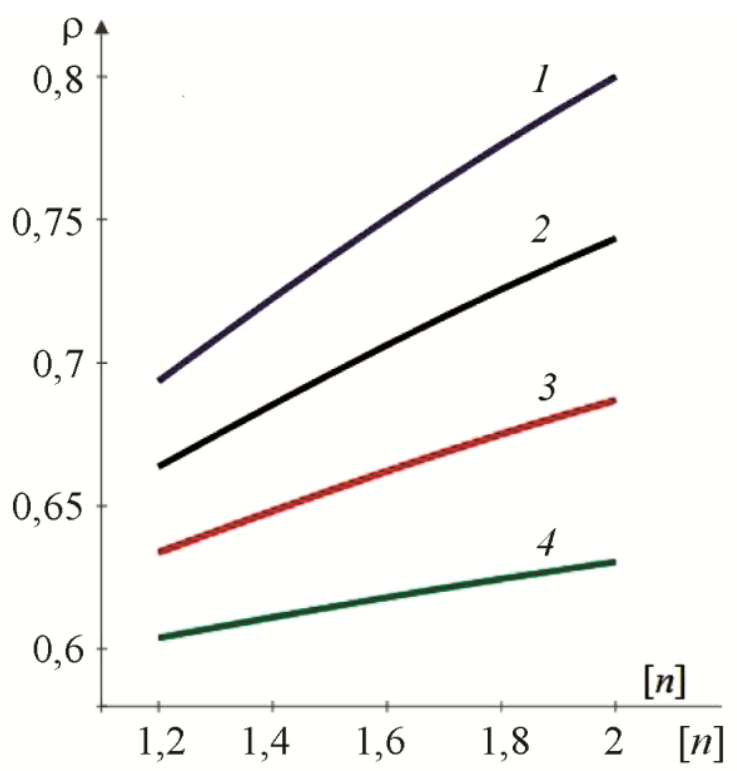

$a$

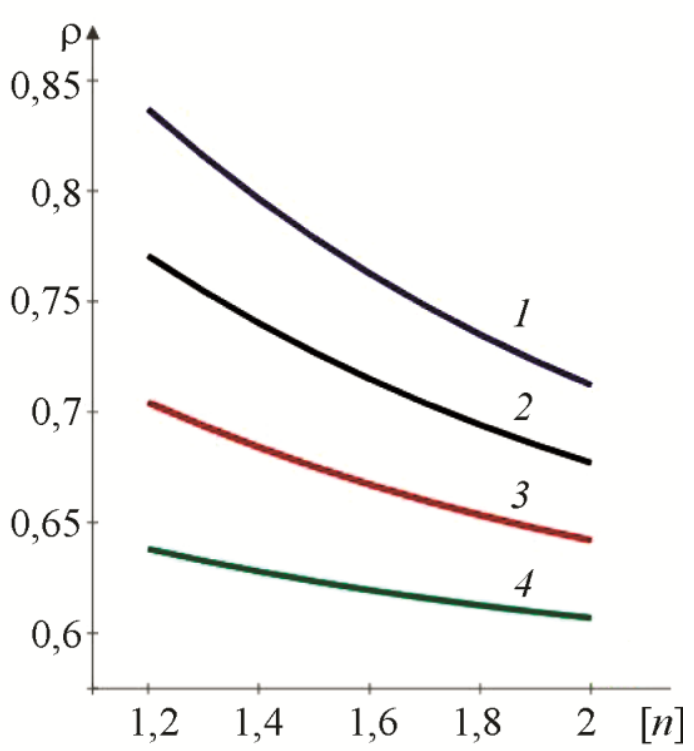

$\sigma$

Рис. 2. Зависимость коэффициента запаса прочности $[n]$ и коэффициента корреляции $\rho$ при изменяющихся значениях коэффициентов корреляции $\rho_{B}(a)$ и $\rho_{\sigma}(\sigma)$.

Параметры линий $1,2,3,4$ указаны в тексте

Таким образом, расчеты по формуле (25) показывают существенное влияние параметров $k_{\sigma}, k_{w}, \rho_{\sigma}, \rho_{B}$ на вычисление плотностей распределения микроструктурных распределений случайных функций прочности (8) и расчетов повреженности (11) для различных ансамблей точек по формулам $(15,16,18,21)$.

\section{8. Зависимость запаса прочности от поврежденности микроструктуры}

Рассмотрим числовые примеры расчета критической поврежденности в двух, трех и четырех точках в зависимости от параметров распределения функции прочности микроструктуры и коэффициента запаса прочности. Пусть вычислены значения коэффициента вариации $k(23)$ и коэффициента корреляции $\rho(25)$ случайной функции прочности. Используя многомерные плотности распределений $(14,17,21)$, вычисляем в каждом случае относительную микроструктурную поврежденность с помощью интегралов $(15,16,22)$. На рис. 3 приведены результаты расчета поврежденности $q([n])$ в зависимости от запаса прочности $[n]$ при $\rho=0,8, k=0.3$.

Свойства микроструктуры материала влияют на запас прочности и критическую поврежденность. Увеличение запаса прочности $[n]$ соответствует уменьшению критической поврежденности $q$, допускаемой при работе конструкции. На запас прочности влияет также коррелированность характеристик микроструктуры при исследовании ансамбля точек. При одинаковом коэффициенте запаса прочности вероятность разрушения в двух точках выше, чем в трех и четырех точках. С возрастанием запаса прочности вероятность разрушения уменьшается для каждого ансамбля точек. 


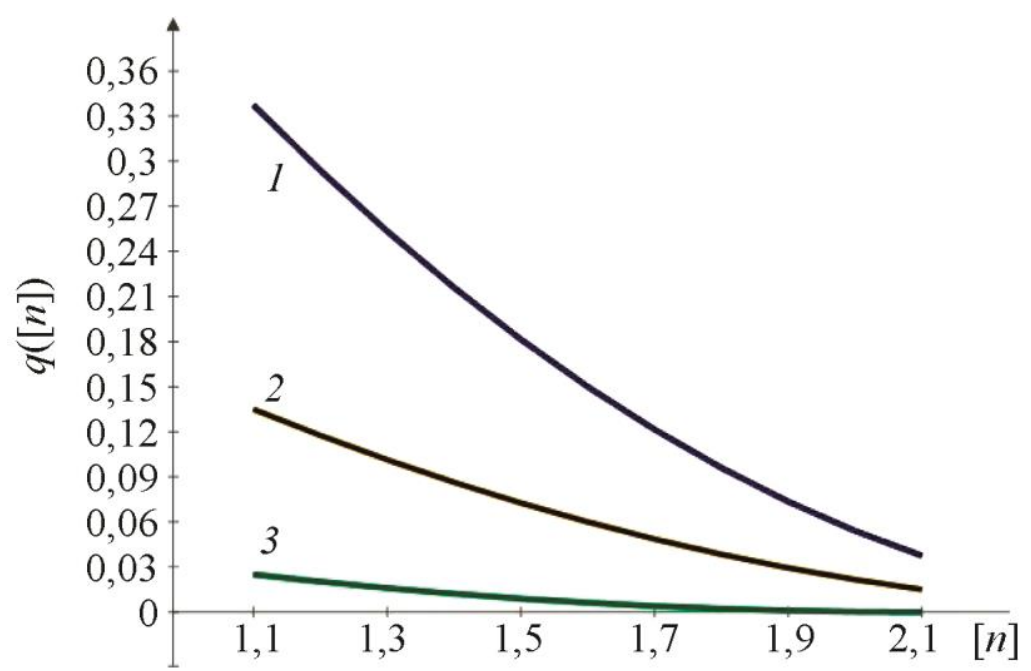

Рис. 3. Зависимость коэффициента запаса прочности $[n]$ и поврежденности $q([n])$ в двух точках (2), трех точках (3) и четырех точках (4)

\section{9. Заключение}

Разработана математическая модель для расчета запаса прочности деформируемых структурно-неоднородных материалов. Оцениваются вероятности возникновения зон разрушения для материалов со случайными свойствами элементов микроструктуры. Используется условие прочности, содержащее корреляционные функции микроструктурных напряжений. Конфигурация множества точек влияет на структуру корреляционных матриц многомерных нормальных законов распределения условия прочности. Показано влияние свойств микроструктуры и параметров распределения напряжений на уровень поврежденности и запаса прочности для одновременного разрушения в двух, трех и четырех элементах микроструктуры материала. Разработанные методы могут быть использованы для неразрушающего контроля конструкционных материалов.

\section{Литература}

1. Yokobori T. An Interdisciplinary Approach to Fracture and Strength of Solids. - Groningen : Wolters-Noordhoff Scientific LTD, 1968.

2. Вильдеман В. Э., Соколкин Ю. В., Ташкинов А. А. Механика неупругого деформирования и разрушения композитных материалов / под ред. Ю. В. Соколкина. - М. : Наука. Физматлит, 1997. - 288 с.

3. Volkova T. A., Volkov S. S. Microstructure damage related to stress- strain curve for grain composites // Theoretical and Applied Fracture Mechanics. - 2009. - Vol. 52, iss. 2. - P. 83-90. DOI: 10.1016/j.tafmec.2008.02.004.

4. Sih G. C. Fracture mechanics in retrospect in contrast to multiscaling in prospect // Proceedings of the 17-th National Conference of Italian Group of Fracture / ed. by A. Finelli and L. Nobile, Bologna, June 16-18, 2004. - P. 15-37.

5. Trusov P. V., Volegov P. S., Yanz A. Yu. Two-scale models of polycrystals: Evaluation of validity of Ilyushin's isotropy postulate at large displacement gradients // Physical Mesomechanics. - 2016. - Vol. 19, no. 1. - P. 21-34. - DOI: 10.1134/S1029959916010033.

6. Micromechanisms of Deformation and Fracture in a VT6 Titanium Laminate under Impact Load / N. S. Surikova, V. E. Panin, L. S. Derevyagina, R. Ya. Lutfullin, E. V. Manzhina, A. A. Kruglov, A. A. Sarkeeva // Phys. Mesomech. - 2015. - Vol. 18, no. 3. - P. 250-260. DOI: $10.1134 /$ S1029959915030091. 
7. Microstructure and properties of low-carbon weld steel after thermomechanical strengthening / V. M. Schastlivtsev, T. I. Tabatchikova, I. L. Yakovleva, S. Yu. Klyueva, A. A. Kruglova, E. I. Khlusova, V. V Orlov // The Physics of Metals and Metallography. - 2012. Vol. 113, no. 5. - P. 480-488. - DOI: 10.1134/S1029959915030091.

8. The Structural State and Properties of a Deposited Coating for An Internal Combustion Engine Valve / N. B. Pugacheva, T. M. Bykova, E. B. Trushina, I. Yu. Malygina // Diagnostics, Resource and Mechanics of materials and structures. - 2018. - Iss. 5. - P. 74-85. DOI: 10.17804/2410-9908.2018.5.074-085. - URL: http://dream-journal.org/issues/2018-5/20185_186.html (accessed 30.11.2019).

9. Зайцев А. В. Моментные функции второго порядка случайной структуры однонаправленно армированных волокнистых композитов // Вестник УГТУ-УПИ. Механика микронеоднородных материалов и разрушение : сборник научных трудов. - Екатеринбург : УГТУ-УПИ, 2006. - № 11 (82). - С. 161-167.

10. A Computational Model of V95/sicp (7075/ Sicp) Aluminum Matrix Composite Applied to Stress-Strain State Simulation under Tensile, Compressive and Shear Loading Conditions / S. V. Smirnov, A. V. Konovalov, M. V. Myasnikova, Yu. V. Khalevitsky, A. S. Smirnov, A. S. Igumnov // Diagnostics, Resource and Mechanics of materials and structures. - 2017. Iss. 6. - P. 16-27. - DOI: 10.17804/2410-9908.2017.6.016-027. - URL: http://dreamjournal.org/issues/2017-6/2017-6_133.html (accessed 14.10.2019).

11. Scientific Basis for Cold Brittleness of Structural BCC Steels and Their Structural Degradation at Below Zero Temperatures / V. E. Panin, L. S. Derevyagina, M. P. Lebedev, A. S. Syromyatnikova, N. S. Surikova, Yu. I. Pochivalov, B. B. Ovechkin // Phys. Mesomech. 2017. - Vol. 2 (2). - P. 125-133. - DOI: 10.1134/S1029959917020023.

12. Structure and Fatigue Durability of 09mn2si Pipe Steel after Long-Term Operation in Far North Conditions / S. V. Panin, P. O. Marushchak, I. V. Vlasov, A. V. Eremin, A. V. Byakov, A. S. Syromyatnikova, R. Stankevich // Diagnostics, Resource and Mechanics of materials and structures. - 2017. - Iss. 4. - P. 81-85. - DOI: 10.17804/2410-9908.2017.4.081-085. URL: http://dream-journal.org/issues/2017-4/2017-4_164.html (accessed 14.10.2019).

13. A Method for Experimental Investigation of Degradation Processes in Materials [Electronic resource] / V. I. Mironov, I. G. Emelyanov, D. I. Vichuzhanin, I. S. Kamantsev, V. V. Yakovlev, D. A. Ogorelkov, L. M. Zamaraev // Diagnostics, Resource and Mechanics of materials and structures. - 2019. - Iss. 2. - P. 16-27. - DOI: 10.17804/2410-9908.2019.2.016-027. URL: http://dream-journal.org/issues/2019-2/2019-2_246.html (accessed 30.11.2019).

14. Митропольский А. К. Техника статистических вычислений. - Москва : Наука, 1971. $576 \mathrm{c}$.

15. Volkova T. A., Volkov S. S. Microstructure damage at ensemble of points for grain composites // Theoretical and Applied Fracture Mechanics. - 2010. - Vol. 54, iss. 3. - P. 149-155. DOI: 10.1016/j.tafmec.2010.10.010.

16. Мак Лин Д. Механические свойства металлов / пер. с англ. Л. И. Миркина ; под ред. Я. Б. Фридмана. - Москва : Металлургия, 1965. - 431 с. 